\title{
Displacement and visualization of point symbols based on spatial distribution characteristics
}

\author{
Haipeng Liu ${ }^{\text {a, b }}$, Yi Long ${ }^{\mathrm{a}, \mathrm{b}} *$, Yi Zheng ${ }^{\mathrm{a}, \mathrm{b}}$ \\ ${ }^{a}$ School of Geography Science, Nanjing Normal University, Nanjing, 210023, China,liu6238819@gmail.com \\ ${ }^{b}$ Key Laboratory of Virtual Geographic Environment (Nanjing Normal University), Ministry of Education, Nanjing, 210023, China \\ * Corresponding author
}

Keywords: real-time cartographic generalization, point data generalization, POI, displacement

\begin{abstract}
:
In WEB2.0 environment, the number of map-based mashups which display user-led POI data keeps increasing. When the cartographic processing of these map mashups is lacking, the display of the POI data showed on the maps are quite unsatisfactory because of the overlapping of symbols.
\end{abstract}

At present, some widely used methods commonly use selection and simplification operations based on a quadtree data structure, which can get a good result in the small and medium scales in which users mainly focus on the distribution characteristics and the density difference of POI, but will lose a lot of information in the large scales in which users mainly focus on the specific location and detailed information of the data. For example, two hotels with the same size will retain only one symbol after using selection or simplification operation although in the large scale if they are adjacent to each other, which will bring trouble to users when using maps. Displacement is a suitable operation to deal with this situation, however, current displacement methods face the problems of symbol position drift and nevertheless the loss of information in high-density areas.

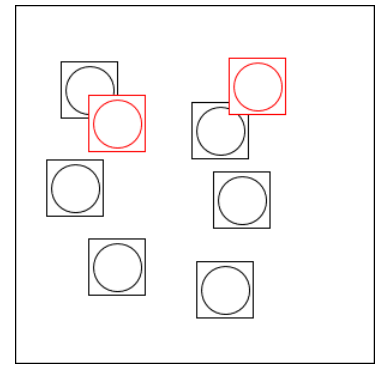

a. Red symbols will be abandoned using selection operation.

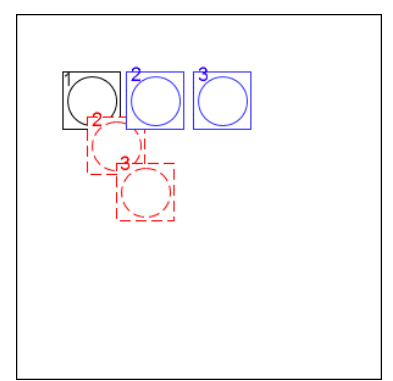

b. Blue symbols drift using displacement operation.

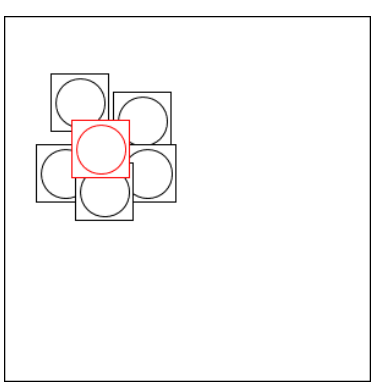

c. Red symbols will be abandoned using displacement operation.

Figure 1. Some problems in current point data generalization methods.

In order to address these problems, this paper proposes a real-time POI visualization algorithm combining the characteristics of traditional quadtree data structure and the advantages of an improved displacement operator.

The main idea behind the algorithms can be described with the following steps:

1. Extract the feature points which can represent the distribution of point groups through quadtree grid.

2. Determine the points to be processed according to map load.

3. Establish a global distance table for the two sets of data above.

4. Detect conflict and displace points according to the order of the distance between the two groups of data.

At last the method is tested on some datasets in the experiment. The result shows that the proposed method can improve the effect of map expression and meet the requirement of real-time processing in the large scales. 


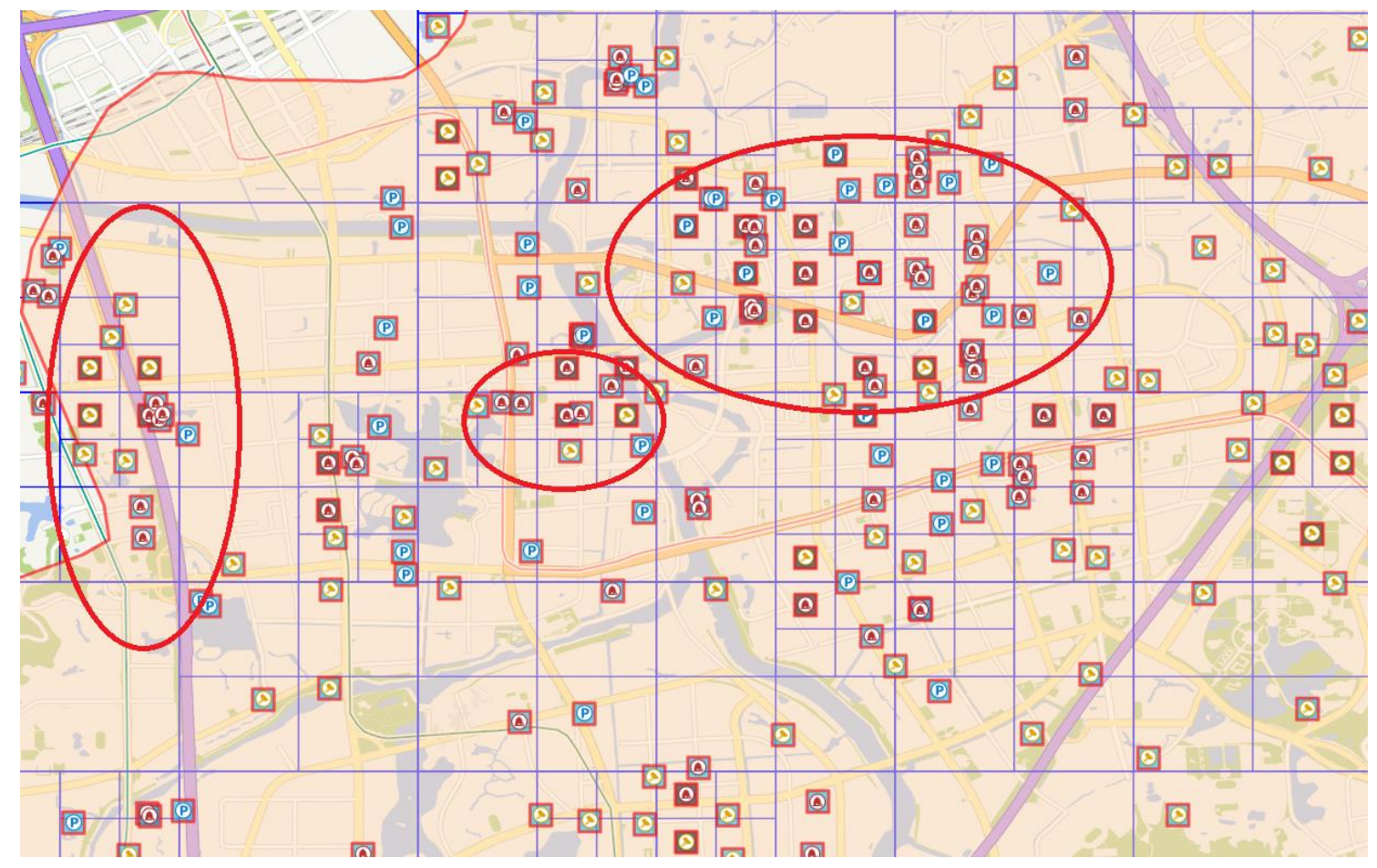

Figure 2. Before processing: overlapping of symbols needs to be resolved.

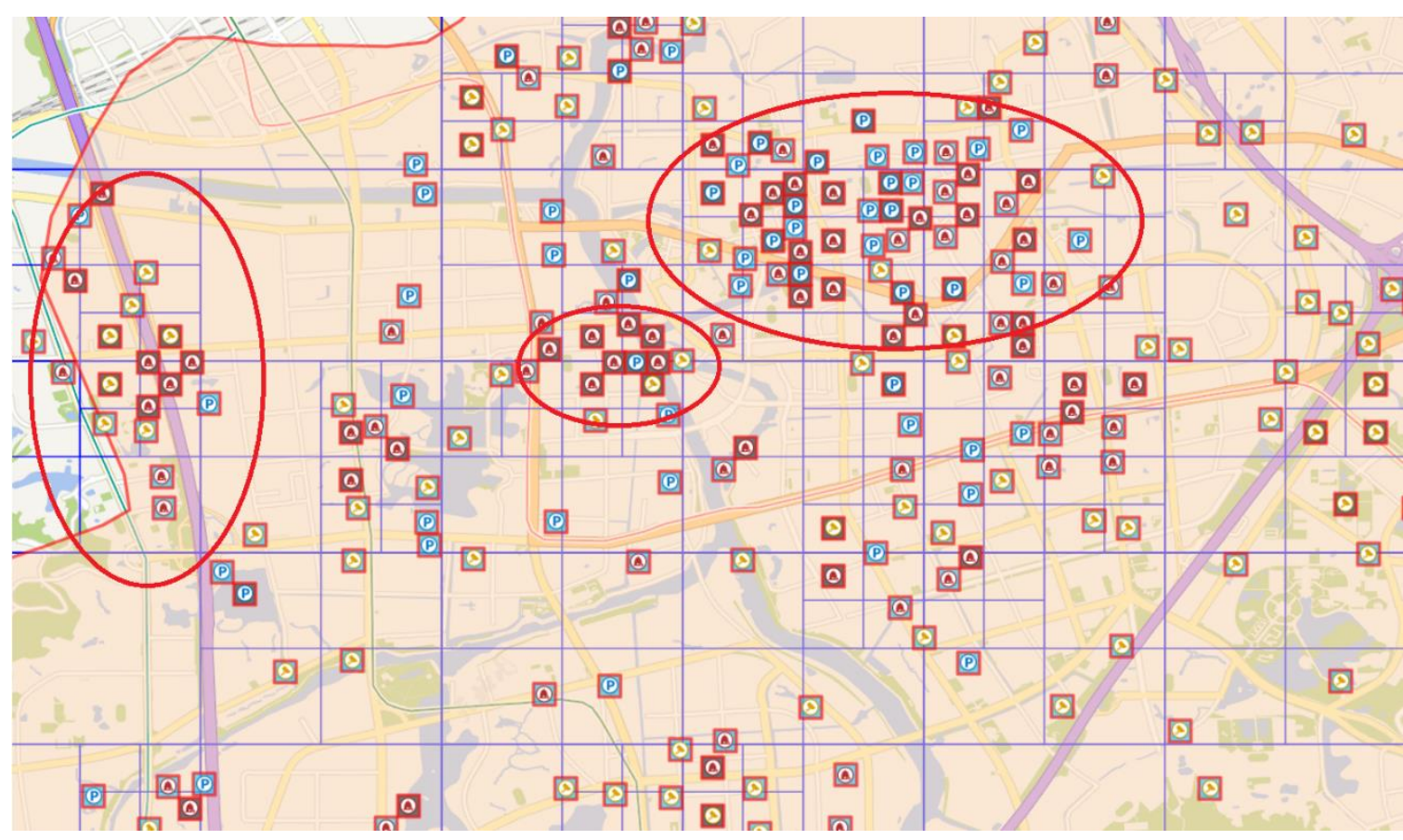

Figure 3. After processing: all the symbols are held without overlapping even in high-density areas, what's more, the spatial distribution characteristics of points have not been changed. 